Real Analysis Exchange

Vol. 24(2), 1998/9, pp. 843-844

Tamás Keleti, *Department of Analysis, Eötvös Loránd University, Rakoczi

ut 5, Budapest, H-1088, Hungary; e-mail: elek@cs.elte.hu

\title{
A 1-DIMENSIONAL SUBSET OF THE REALS THAT INTERSECTS EACH OF ITS TRANSLATES IN AT MOST A SINGLE POINT
}

\begin{abstract}
We construct a compact subset of $\mathbf{R}$ with Hausdorff dimension 1 that intersects each of its non-identical translates in at most one point. Moreover, one can make the set to be linearly independent over the rationals.
\end{abstract}

In 1984 P. Mattila [2] constructed compact subsets $A$ and $B$ of $\mathbf{R}$ with Hausdorff dimension 1 such that the intersection of $A$ and any translate of $B$ contains at most one point. In this note we show that - if we allow only non-identical translations - one can also have $A=B$.

We call a set of 3 or 4 real numbers $x_{1}<x_{2} \leq x_{3}<x_{4}$ a rectangle if $x_{2}-x_{1}=x_{4}-x_{3}$.

Note that a set intersects each of its translates in at most one point if and only if the set does not contain a rectangle. (Here and in the sequel by set we will always mean a subset of $\mathbf{R}$ and by translate a non-identical translate.)

Theorem 1. There exists a compact set with Hausdorff dimension 1 that intersects each of its translates in at most one point.

Proof. Let $\delta_{m}=1 /\left(6^{m-1} m !\right)$. We inductively define compact sets $A_{m}$ as disjoint unions of closed intervals $\left[n_{i_{1} \ldots i_{m}} \delta_{m},\left(n_{i_{1} \ldots i_{m}}+1\right) \delta_{m}\right]$ for $1 \leq i_{k} \leq k$, $1 \leq k \leq m$. We will denote by $I_{1}^{m}, I_{2}^{m}, \ldots, I_{m !}^{m}$ the intervals of $A_{m}$ and by $\left(J_{1}, J_{2}, \ldots\right)$ the sequence $\left(I_{1}^{1}, I_{1}^{2}, I_{2}^{2}, I_{1}^{3}, \ldots, I_{3 !}^{3}, \ldots\right)$.

\footnotetext{
Key Words: Hausdorff dimension, translation, linearly independent, intersection

Mathematical Reviews subject classification: $28 \mathrm{~A} 78$

Received by the editors June 8, 1998

* This research was done while the author was visiting the University College London having a Royal Society/NATO Postdoctoral Fellowship award.
} 
Let $n_{1}=0$. (Then $A_{1}=I_{1}^{1}=J_{1}=[0,1]$.) Assume that $A_{1}, \ldots, A_{m}$ have already been defined. If $n_{i_{1} \ldots i_{m}} \delta_{m} \notin J_{m}$ then let

$$
n_{i_{1} \ldots i_{m} i}=6(m+1) n_{i_{1} \ldots i_{m}}+6 i-6 \quad(i=1, \ldots, m+1),
$$

and if $n_{i_{1} \ldots i_{m}} \delta_{m} \in J_{m}$ then let

$$
n_{i_{1} \ldots i_{m} i}=6(m+1) n_{i_{1} \ldots i_{m}}+6 i-3 \quad(i=1, \ldots, m+1) .
$$

Thus

$$
\left[n_{i_{1} \ldots i_{m} i} \delta_{m+1},\left(n_{i_{1} \ldots i_{m} i}+1\right) \delta_{m+1}\right] \subset\left[n_{i_{1} \ldots i_{m}} \delta_{m},\left(n_{i_{1} \ldots i_{m}}+1\right) \delta_{m}\right]
$$

for $i=1, \ldots, m+1$, which means that the intervals of $A_{m+1}$ are contained in the intervals of $A_{m}$.

Let $A=\cap_{l=1}^{\infty} A_{m}$. Then $A$ has Hausdorff dimension 1, cf. [1] Example 4.6. Hence, by our previous remark, it is enough to show that $A$ does not contain a rectangle.

Let $x_{1}<x_{2} \leq x_{3}<x_{4}$ be points of $A$. Take an $m$ such that $\delta_{m}<x_{2}-x_{1}$. Then if $x_{1} \in I_{j}^{m}=J_{M}$ then none of $x_{2}, x_{3}$ and $x_{4}$ is in $I_{j}^{m}$. Thus, when we defined $A_{M+1}$, we used (2) for defining the interval that contains $x_{1}$ and (1) for defining the intervals that contain $x_{2}, x_{3}$ and $x_{4}$. This implies that $x_{1}$ is of the form $\left(6 N_{1}+3\right) \delta_{M}+\varepsilon_{1}$ but $x_{2}, x_{3}$ and $x_{4}$ are of the form $6 N_{j} \delta_{M}+\varepsilon_{j}$, where $N_{1}, \ldots, N_{4}$ are integers and $0 \leq \varepsilon_{i} \leq \delta_{M}$ for $i=1, \ldots, 4$. Thus $x_{2}-x_{1} \neq$ $x_{4}-x_{3}$, which means that $\left(x_{1}, x_{2}, x_{3}, x_{4}\right)$ is not a rectangle.

Remark 2. Slightly modifying the above construction (by replacing 6 with a slowly increasing sequence of even numbers) one can also get a compact set with Hausdorff dimension 1 which is linearly independent over the rationals. (The existence of a linearly independent perfect set is well known, even in any non-discrete locally compact abelian group, see e. g. [3].)

\section{References}

[1] K. Falconer, Fractal Geometry, John Wiley \& Sons, 1990.

[2] P. Mattila, Hausdorff dimension and capacities of intersections of sets in n-space, Acta Math., 152 (1984), 77-105.

[3] W. Rudin, Fourier Analysis on Groups, Interscience Publishers, New York - London, 1962. 